\title{
Kernos
}

Revue internationale et pluridisciplinaire de religion grecque antique

13 | 2000

Varia

\section{Mitografia di Cassandra: un recente contributo}

\section{Sabina Mazzoldi}

\section{CpenEdition \\ Journals}

\section{Édition électronique}

URL : http://journals.openedition.org/kernos/1305

DOI : 10.4000/kernos.1305

ISSN : 2034-7871

\section{Éditeur}

Centre international d'étude de la religion grecque antique

\section{Édition imprimée}

Date de publication : 1 janvier 2000

ISSN : 0776-3824

\section{Référence électronique}

Sabina Mazzoldi, « Mitografia di Cassandra: un recente contributo», Kernos [En ligne], 13 | 2000, mis en ligne le 21 avril 2011, consulté le 21 septembre 2020. URL : http://journals.openedition.org/kernos/ 1305 ; DOI : https://doi.org/10.4000/kernos. 1305 
contraire, à partir des mêmes sources et avec les mêmes règles de la critique historique. De là une divergence essentielle : pour Steuernagel, les Étrusques ne sont pas rebutés à l'idée du sacrifice humain; pour moi, ils le sont tout autant que les Grecs et les Romains ${ }^{1}$ et ainsi de suite. Pour pallier ces divergences d'interprétation, nullement irréversibles, une réflexion commune s'impose, incluant évidemment l'historiographie toujours plus pernicieuse qu'on ne le pense, et à laquelle doivent participer des représentants de toutes les disciplines incriminées, historiens et historiens des religions, anthropologues et archéologues. Il y a du pain sur la planche, et tant que ce travail ne sera pas effectué, tout comparatisme au niveau méditerranéen sera voué au plus cuisant des échecs ${ }^{2}$.

\title{
Mitografia di Cassandra: un recente contributo ${ }^{3}$
}

\author{
Sabina Mazzoldi (Università di Urbino)
}

Nell'ultimo decennio la figura di Cassandra è stata argomento di numerose dissertazioni di dottorato, in particolare, ma non solo, nelle università tedesche ${ }^{4}$. Una tale fioritura di studi, giustificata dalla poliedricità del personaggio stesso e dalla sua grande, ininterrotta fortuna nella cultura europea, è stata indubbiamente stimolata dalla pubblicazione dell'importante e fortunato romanzo di Christa Wolf, Kassandra (1983). Molti di questi lavori, infatti, prendono sì le mosse dai testi classici greci e latini, fonti essenziali per la definizione dei caratteri primi del personaggio, ma focalizzano l'indagine sul suo Fortleben nella letteratura medievale, moderna e contemporanea, con approcci non tanto filologico-letterari, quanto di tipo filosofico e/o psicologico, oppure pertinenti alla teoria della letteratura.

Nella sua monografia, anch'essa sviluppo di una tesi di dottorato, la Neblung ritorna, invece, ad una indagine di stampo tradizionale. L'A. procede ad un sistematico esame di tutte le fonti letterarie, greche e latine, relative al personaggio di Cassandra, ordinate cronologicamente: epos e lirica (I), tragedia greca (II), lettera-

\footnotetext{
1 Nous laisserons les pauvres Carthaginois de côté pour le moment.

2 A titre d'exemple, l'essai malheureux de J.E. Miller, The Western Paradigm. Greek and Hebrew Traditions, San Francisco/Londres/Behtesda, 1996.

3 D. Neblung, Die Gestalt der Kassandra in der Antiken Literatur (Diss. Berlin 1996), Stuttgart und Leipzig, 1997.

4 Cf. B. FeICHTINGER, Kassandra und die Dichter. Studien zu Mythographie antiken Autoren, Diss. Salzburg, 1988; T. Epple, Der Aufstieg der Untergangsseberin Kassandra. Zum Wandel ibrer Interpretation vom 18. Jabrbundert bis zur Gegenwart, Diss, Wurzburg, 1992 (pubbl. 1993); S. Müller, Kein Brautfest zwiscben Menscben und Göttern. Kassandra-Mytbologie im Licbte von Sexualität und Wabrbeit, Diss. Dusseldorf, 1993 (pubbl. Köln, 1994); S. Jentgens, Kassandra. Spielarten einer literariscben Figur, Diss. Osnabrück, 1994 (pubbl. Hildesheim/Zürich, 1995); meno recente K. LEDERGERBER, Kassandra. Das Bild der Prophetin in der antiken und insbesondere in der alteren abendländiscben Dicbtung, Diss. Freiburg, 1951; $c f$. anche A. Brault Pascal, Prophetess Doomed. Cassandra and the Representation of Trutb, Diss. NewYork, 1990; S. MazzoLDI,

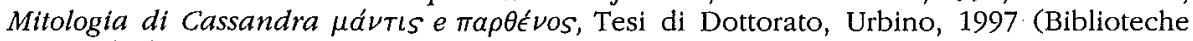
Nazionali di Firenze e Roma).
} 
tura ellenistica (III), tragedia repubblicana (IV), Cicerone e letteratura augustea (V), Seneca (VI), Seconda Sofistica (VII), autori greci e latini dei secoli 2-5 (VIII), epici tardi (IX). Ogni capitolo si compone di una sezione analitico-descrittiva, con una trattazione talora dettagliata, talaltra piuttosto cursoria delle fonti e con brevi e sporadici approfondimenti di carattere filologico, il più delle volte relegati nelle note, e di un paragrafo di "risultati". Completano il volume una serie di utili indici, che rendono agevole la consultazione: 1 . indice dei motivi del mito (dono della veggenza, Aiace, Agamennone) con rimando ai singoli passi; 2 , elenco dei passi (con tutti i testi noti, in ordine alfabetico per autore, che si riferiscono a Cassandra); 3 . indice dei luoghi citati, con rimando alle pagine del volume.

Sebbene l'A. dichiari di non aver voluto occuparsi dei diversi aspetti di Cassandra isolatamente (bellezza, verginità, veggenza, relazione con Aiace, relazione con Agamennone, morte), in quanto si tratta di una figura complessa, di cui i singoli elementi stanno in un rapporto di interdipendenza, in realtà proprio tali aspetti figurano come titolo di numerosi paragrafi, che talvolta appaiono slegati. Nell'esame delle fonti più tarde l'attenzione è volta soprattutto ad individuare linee di continuità e margini di innovazione rispetto alla tradizione prece dente.

L'elenco delle fonti è esaustivo ${ }^{1}$ e indubbiamente aggiornato rispetto al datato volume della Davreux ${ }^{2}$, che fino ad ora rappresentava il contributo più completo sulla profetessa troiana; l'A. può includere nella sua trattazione anche scoperte papiracee relativamente recenti ${ }^{3}$. La scelta di non considerare le fonti figurative pregiudica tuttavia i risultati stessi del lavoro, non tanto perché vi fosse necessità di una loro elencazione, reperibile altrove ${ }^{4}$, quanto perché esse offrono un sostanziale contributo nella ricostruzione di quello "sviluppo letterario della figura di Cassandra", che vuole essere lo scopo primario del lavoro della Neblung (p. 4). È il caso, ad esempio, dell'episodio mitico dell'oltraggio di Aiace nel tempio di Atena, che ebbe enorme fortuna nella produzione iconografica (dalla ceramica attica a figure nere e rosse, a quella italiota, fino ai manufatti di ambiente etrusco e romano) e che, proprio grazie ad essa, può essere ricostruito in una prospettiva diacronica, nelle diverse interpretazioni che ne hanno dato i poeti ${ }^{5}$. Analogamente, la Tabula Iliaca appare fondamentale per stabilire la presenza di Cassandra nella sua qualità di profetessa già nella perduta Ilias Parva

1 Manca tuttavia la dedica della targhetta in bronzo, ritrovata a Dodona e risalente al IV-III sec., in cui un tale Agatone, figlio di Echephylos, originari dell'isola di Zacinto, rivendica la discendenza da KASSANDRA la troiana ( $c f$. C. Carapanos, Dodone et ses ruines, Paris, 1878 , t. I p. 196-199, t. II pl. XXII). L'A. avrebbe potuto anche menzionare Ellanico, Troika, fr. 202, 3 Caerols Pérez (P.Univ. Giss. 4, $40=2810$ Pack $^{2}$ ), dove compare l'espressione ásıklns tô Alavtos, probabile testimonianza della presenza, nell'opera, dell'episodio mitico dell'oltraggio di Aiace a Cassandra.

2 J. Davreux, La légende de la prophétesse Cassandre d'après les textes et les monuments, Liège 1942.

3 Cf. p.es. P.Oxy. 2746 (TrGF II F adesp. 649); P.Oxy. 3650 (bypothesis dell'Alessandro di Euripide); P.Köln 2021 e P.Oxy. 2303 (Alceo fr. 298 Voigt); P.Oxy. 3830 fr, 1 (Mytbograpbus Homericus). Nello "Stellenregister" (p. 259) il Tebtunis Papyrus di Ditti Cretese figura come n. 266 invece che n. 268.

4 Oltre al repertorio presente in Davreux, p. 97-236, si dispone di elenchi aggiornati nel LIMC, s.v. Agamemnon (vol. I, p. 270-72), Aias II (vol. I, p. 336-51), Alexandros (vol. I, p. 494-529), Athena (vol. II, p. 966-68), Kassandra I (vol. VII Addenda, p. 956-70).

5 Cf. S. Mazzoldi, Cassandra, Aiace e lo Goavov di Atena, in QUCC, 55 (1997), p. 7-22.

6 Come è noto, è ancora discusso se nei poemi omerici Cassandra sia $\mu d \dot{v}$ Tis; potrebbe esservi un'allusione alle sue doti di profetessa in $I l$., XXIV, 697-706, ma a mio 
nel quinto riquadro della banda inferiore del basamento, tutta dedicata a questo poema del Ciclo, l'eroina è rappresentata accanto al cavallo di legno, che viene trascinato verso le porte di Troia ${ }^{1}$. Si tratta di un episodio che ebbe larghissima fortuna, tanto in ambito letterario quanto nella produzione figurativa, e la testimonianza è importante in quanto permette di affermare con relativa certezza che, nell'ambito dei poemi del Ciclo, il personaggio di Cassandra era $\mu a ́ v \tau ا S$ non solo nei Cypria ${ }^{2}$.

Altrettanto discutibile sembra la scelta di escludere l'ambito religioso (p. 5): un approfondimento delle fonti in questa direzione appare fondamentale. In primo luogo il fenomeno della divinazione, così come trova riflesso nella produzione letteraria, è comprensibile soltanto alla luce del suo radicamento in precisi contesti storico-religiosi. In altre parole, la figura letteraria di Cassandra $\mu a ́ v T ı s$ è fortemente condizionata dall'esperienza che $i$ diversi autori, calati in un preciso ambito culturale e religioso, potevano avere della divinazione, in particolare di quella estatica, e di figure reali come la Pizia o indovini di modello sibillino $^{3}$. La Cassandra dell' Agamennone eschileo, ad esempio, è forse la più efficace rappresentazione del $\mu a ́ v T i s$ del $V$ secolo nell'atto di profetizzare ${ }^{4}$ e la più compiuta espressione del suo ruolo nella comunità ateniese della polis; ruolo che già appare compromesso nelle Troiane euripidee. Qui l'impiego omogeneo e sistematico del lessico bacchico è spia di una scelta programmatica e provocatoria: Euripide rinuncia al vocabolario mantico e lo sostituisce con quello bacchico non perché voglia connotare l'estasi in termini peggiorativi, come follia (p. 44, 54), o in termini di oscurità e enigmaticità (p. 48, 54) (ciò sarebbe vero se tale lessico figurasse usato soltanto in riferimento $a$ Cassandra e non anche sfruttato $d a$ Cassandra stessa). La sua caratterizzazione come baccante esplicita l'incolmabile distanza tra la $\mu$ ávTıS e i suoi ascoltatori, la sua estraneità e la sua emarginazione rispetto alla comunità, tanto dei vincitori, i Greci, quanto dei vinti, i Troiani. Tale sviluppo nella configurazione di Cassandra veggente/ menade trova interessanti conferme nella produzione figurativa.

Ma la considerazione dell'aspetto storico-religioso nell'esame delle fonti relative a Cassandra è fondamentale anche da un altro punto di vista: la sua figura letteraria o, meglio, le interpretazioni letterarie del suo mito appaiono strettamente connesse ai culti presenti in vari luoghi della Grecia (Argolide, Laconia ${ }^{5}$,

avviso il riferimento sarebbe dovuto essere più esplicito e tali doti emergerebbero anche in altri passi del poema. La Neblung (p. 7), citando un articolo di Treu, afferma "non è provabile come non è contestabile che Omero conosceva Cassandra come profetessa".

1 Cf. A. SAdurska, Les Tables lliaques, Warszawa, 1964, p. 28, 30.

2 Cf. Poet. Ep. Gr. I, 39, 9-11 Bernabé.

3 Appare significativa a questo riguardo l'identificazione di Cassandra, nell'Alessandra di Licofrone, con la Sibilla (v, 1464-1465), che a sua volta è talora identificata con Artemide ( $c f$. Paus., $\mathrm{X}, 12,2$; ciò getta luce sui rapporti tra Artemide e la mantica, del tutto inesistenti secondo la Neblung, p. 106): tra IV e III sec. a.C. vi è una vera e propria proliferazione di Sibille e nel milieu culturale ellenistico la profezia ex eventu acquista un significato storico-politico fondamentale.

4 Da questo punto di vista la resa eschilea sembra corrispondere al quadro antropologico del fenomeno della divinazione, che possiamo ricostruire attraverso fonti di genere diverso. A questo riguardo va precisato che le visioni di Cassandra, come la sua glossolalia, trovano riscontro in esempi 'storici' di divinazione estatica, e non vanno interpretate, nell'economia della tragedia eschilea, come mezzo, utilizzato da Apollo, per impedire la chiarezza della profezia (così invece l'A., p. 28).

5 Complesso e per molti aspetti ancora irrisolto è, p.es., il problema dell'identificazione tra la dea Alessandra di Amicle e Cassandra, cui la Neblung accenna sommariamente (p. 191): quando e su che basi avvenne tale identificazione? E fu essa avvantaggiata 
Locride) e della Magna Grecia (Daunia); sono essi, nell'inscindibile binomio mito-rito, che concorrono alla ricostruzione di quel "mito fondamentale", reale solo nelle sue varianti, che, secondo le più moderne prospettive degli studi di mitologia, "fu in grado di soddisfare le ricezioni e le aspettative" ${ }^{1}$. Da questa prospettiva le connessioni tra la funzione profetica di Cassandra e quella sacerdotale meriterebbero una particolare indagine ${ }^{2}$.

Degne di approfondimento sono anche le valenze antropologiche (oltre che, ovviamente, drammatiche all'interno dei singoli contesti poetici) del suo non essere creduta: l'A. considera, soprattutto nell'economia di ogni singola tragedia, i risvolti letterari della condanna che pesa sulla profetessa, ma non approfondisce la tematica, che si rivela interessante anche sotto il profilo semiotico, come emerge, per esempio, da un confronto tra l'Agamennone di Eschilo e l'Alessandra di Licofrone relativamente al rapporto emittente/destinatario del messaggio oracolare/pubblico (il pubblico del teatro ateniese o quello letterato e 'libresco', forse la corte tolemaica stessa, presupposto dal poeta ellenistico).

Anche alcune conclusioni risultano non del tutto condivisibili e l'analisi lessicale dei testi avrebbe meritato maggior approfondimento ${ }^{3}$. A proposito dell'incredibilità delle profezie di Cassandra, la Neblung distingue, giustamente, due procedimenti attraverso i quali gli autori la comunicano al pubblico: alcuni caratterizzano la lingua usata come metaforica e oscura e quindi il messaggio, ancor prima che incredibile, risulta incomprensibile agli ascoltatori; altri attribuiscono alla veggente profezie così contrastanti con la situazione, che appaiono inverisimili (p. 234). Se ciò in linea di massima può essere vero, in realtà il quadro si presenta molto più complesso, i gradi di metaforismo e di oscurità della profezia sono vari e l'A. non sembra tenere sufficientemente conto della disposizione d'animo e della disponibilità all'ascolto dei destinatari delle profezie stesse. Nel caso dell'Agamennone di Eschilo, ad esempio, collocato dalla Neblung nella prima categoria, il messaggio oracolare raggiunge livelli di assoluta intelligibilită ${ }^{4}$, ma.è il Coro dei vecchi argivi a non poter riceverlo ed accettarlo, soprattutto quando esso riguarda il suo re $\mathrm{e}^{5}$.

dalla coincidenza di ubicazione tra culto e vicenda eroica dell'uccisione di Cassandra e Agamennone in talune fonti letterarie (Simonide, Stesicoro, Pindaro), oppure, al contrario, fu essa, già avvenuta prima del VI sec., a permettere lo spostamento dello scenario della vicenda dall'Argolide (Omero) in Laconia, dove il culto era localizzato?

1 Cf. H. Blumenberg, Elaborazione del mito, trad. it. Bologna, 1991 [Frankfurt am Main, 1979], p. 219.

2 Tale funzione è indiscutibile, ad esempio, nelle Troiane di Euripide, come rileva opportunamente la NeBlung (p. 52 n. 60), ma è molto probabile che emergesse anche dall'Alessandro, fr. 6 Snell e fr. 64 Snell $\left(=867\right.$ Nauck $\left.^{2}\right)$. Il problema è complesso dal momento che Cassandra si trova ad essere contemporaneamente profetessa e sacerdotessa e se i rapporti tra le due 'professioni' nel mondo greco sono stretti, non sono stati ancora chiariti.

3 Il lessico mantico usato in relazione a Cassandra si presenta molto omogeneo e costante nelle varie fonti. In TrGF II F adesp. 649, 24 la Neblung crede che ăkpav $\gamma \hat{\eta} p u v$ sia il grido di morte di Ettore (p. 95), ma, pur tenendo conto delle difficoltà nell'attribuire le battute, è assai più probabile che si tratti di un riferimento alla voce profetica di Cassandra: in Lyc., Alex., 7, yîpus è la voce della Sfinge imitata da Alessandra e, per quanto

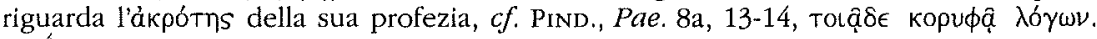

4 Il caso più evidente è il v. 1246 "di Agamennone ti dico che vedrai la morte", cui il Coro reagisce intimando il silenzio.

5 Diversa è la sua disponibilità all'ascolto quando Cassandra profetizza il proprio destino di morte: qui il Coro, spinto dalla pietà, sembra arrivare a capire e a credere $(c f$. p.es. v. 1162). 
Appena accennata è la problematica dei rapporti Cassandra/Agamennone che, nelle varie testimonianze poetiche, appaiono diversamente configurati, probabilmente in funzione di diversi quadri socio-culturali. Se nell'Agamennone di Eschilo è il sacrificio di Ifigenia il motivo determinante per l'uccisione di

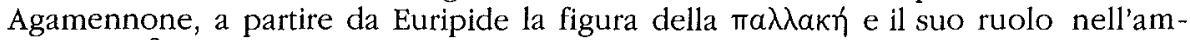
bito dell'ốkos (il cosiddetto tema del 'doppio talamo') risultano problematizzati, il poeta tragico disegna un triangolo amoroso e fa dell'infedeltà di Agamennone e della conseguente gelosia di Clitemestra la vera molla dell'uxoricidio: nelle Troiane Cassandra si può configurare come vendicatrice di Troia in quanto appare causa diretta dell'assassinio di Agamennone. Nella produzione letteraria posteriore l'amore di Agamennone per Cassandra si cristallizza come topos e funge da exemplum; la sua infedeltà, d'altra parte, diventa definitivamente la causa maggiore dell'uxoricidio, mentre il sacrificio di Ifigenia è relegato in posizione marginale.

Sull'impostazione della bibliografia, essenziale e scolastica, si possono avanzare delle riserve: la suddivisione in due sezioni ("Texte" e "Sekundärliteratur") e in numerose sotto-sezioni, che rispecchiano l'organizzazione del lavoro per capitoli, obbliga a un fastidioso gioco di rimandi interni e la rende di non immediata consultazione. Se sono presenti i fondamentali strumenti per l'esegesi dei testi considerati ${ }^{1}$, spiccano per la loro assenza alcuni contributi su Cassandra ${ }^{2}$ e opere su tematiche più generali (divinazione, femminilità), strettamente connesse a questa figura, imprescindibili, a mio avviso, per uno studio approfondito sul personaggio, anche volendo limitarsi a un punto di vista meramente letterario ${ }^{3}$.

In conclusione, la monografia della Neblung si presenta come un esaustivo e comodo repertorio delle fonti relative al personaggio di Cassandra, analizzate in maniera descrittiva ed essenziale, ma non sembra tenere conto delle moderne metodologie di indagine mitologica e risponde solo in parte all'esigenza di rimpiazzare il lavoro della Davreux, obsoleto proprio per il suo impianto generale.

Via Sagginalese 2, I - 50032 Borgo S. Lorenzo (FIRENZE)

e-mail: sagginale@dada.it

1 Eppure mancano, p.es., R. THIEL, Chor und tragische Handlung im 'Agamemnon' des Aischylos, Stuttgart, 1993 (valido strumento per l'interpretazione della scena di Cassandra), N.T. Croally, Euripidean Polemic. The Trojan Women and the Function of Tragedy, Cambridge, 1994 (in cui è analizzato il ragionamento di Cassandra e la sua funzione vendicatrice secondo una prospettiva vicina a quella della Neblung). Fondamentale è poi il recente saggio di S. Timpanaro, Dall'Alexandros di Euripide all'Alexander di Ennio, in $R F I C, 124$ (1996), p. 5-70.

2 Cf., p.es., i saggi di A. Moreau pubblicati in CGITA, 4 (1988), p. 103-114; Kentron, 4 (1988), p. 113-132; Entre bommes et dieux, Paris, 1989 (Ann. littér. de l'Univ. de Besançon, $86)$, p. $145-167$

3 Sarebbe stato utile, talora, un rimando ai classici F.A. Bouché-LeClere, Histoire de la divination dans l'antiquité, 4 vol., Paris 1879-1882, о A. СAquot, M. LeIBovici, La divination, Paris, 1968, o ai recenti, e peraltro fondamentali secondo le più moderne prospettive di indagine mitico-letteraria, Divination et rationalité, Paris, 1974 (che fornisce coordinate essenziali per interpretare il messaggio profetico di Cassandra), R. SEAford, Reciprocity and Rilual, Oxford 1994, ma anche R. ReHM, Marriage to Death. The Conflation of Wedding and Funeral Rituals in Greek Tragedy, Princeton N.J., 1994 e C. Faraone, T. CARPENTER (eds.), The Masks of Dionysos, Ithaca NY, 1993 (numerosi contributi sull'uso del lessico bacchico in tragedia), se non altro per dare un orientamento sugli studi più attuali relativamente a problematiche cui l'A. accenna in maniera piuttosto superficiale. 